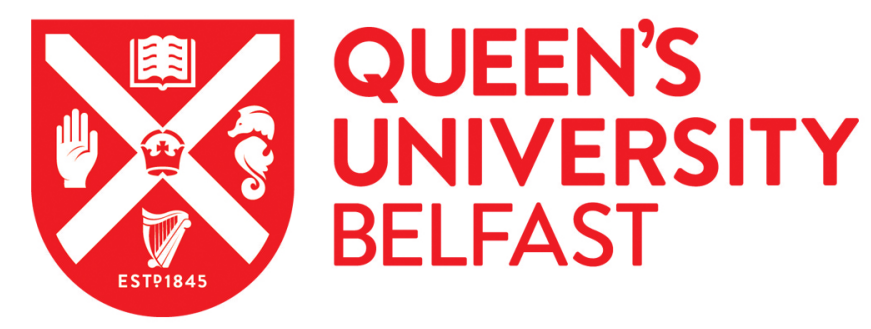

\title{
Long term exposure to a butter rich diet induces mild-to-moderate steatosis in Chang liver cells and swiss albino mice models
}

Nalloor, T. J. P., Kumar, N., Narayanan, K., \& Palanimuthu, V. R. (2017). Long term exposure to a butter rich diet induces mild-to-moderate steatosis in Chang liver cells and swiss albino mice models. Journal of Basic and Clinical Physiology and Pharmacology. https://doi.org/10.1515/jbcpp-2016-0058

Published in:

Journal of Basic and Clinical Physiology and Pharmacology

Document Version:

Publisher's PDF, also known as Version of record

Queen's University Belfast - Research Portal:

Link to publication record in Queen's University Belfast Research Portal

Publisher rights

(c) 2017 by Walter de Gruyter $\mathrm{GmbH}$.

This work is made available online in accordance with the publisher's policies.

\section{General rights}

Copyright for the publications made accessible via the Queen's University Belfast Research Portal is retained by the author(s) and / or other copyright owners and it is a condition of accessing these publications that users recognise and abide by the legal requirements associated with these rights.

Take down policy

The Research Portal is Queen's institutional repository that provides access to Queen's research output. Every effort has been made to ensure that content in the Research Portal does not infringe any person's rights, or applicable UK laws. If you discover content in the Research Portal that you believe breaches copyright or violates any law, please contact openaccess@qub.ac.uk. 
Thomas John Philip Nalloor, Nitesh Kumar, Kasinathan Narayanan and Vasanth Raj Palanimuthu*

\section{Long-term exposure to a butter-rich diet induces mild-to-moderate steatosis in Chang liver cells and Swiss albino mice models}

DOI 10.1515/jbcpp-2016-0058

Received April 14, 2016; accepted October 17, 2016

\section{Abstract}

Background: Butter is one of the widely used fats present in the diet. However, there is no satisfactory study available that evaluates the effect of a high-fat diet containing butter as the principal fat on the development of non-alcoholic fatty liver disease (NAFLD).

Methods: In the present study, butter was used for the development of steatosis in Chang liver cells in an in vitro study and Swiss albino mice in an in vivo study. In vitro steatosis was established, and butter was compared with oleic acid in Chang liver cells using an oil red O (ORO)based colorimetric assay. In the in vivo study, a butter-rich special diet was fed for 15 weeks to mice, who showed no significant change in body weight. The expression pattern of phosphatase and tensin homolog (PTEN) and miR-21 was compared by reverse transcriptase-PCR.

Results and Conclusions: Special diet-fed animals showed downregulated PTEN compared to normal diet-fed animals, while levels of miR-21 remained the same. Elevations in biochemical parameters, viz., triglycerides and liver function tests showed symptoms of onset of NAFLD. Histophathological study of livers of test animals confirmed mild-to-moderate degree of NAFLD.

*Corresponding author: Vasanth Raj Palanimuthu, MPharm, PhD, Associate Professor, Faculty of Pharmacy, AIMST University, Jalan Bedong Semeling 08100, Bedong Kedah Darul Aman, Malaysia; and Pharmaceutical Sciences and Pharmaceutical Biotechnology, China Medical University-Queen's University Belfast Joint College (CQC), Shenyang, Liaoning Province 110122, P.R. China, Phone: 006044298000; 006044298151 (ext: 1284), Fax: 006044298009 , E-mail: vasanth1780@gmail.com

Thomas John Philip Nalloor and Kasinathan Narayanan:

Department of Pharmaceutical Biotechnology, Manipal College of Pharmaceutical Sciences, Manipal University, Manipal 576104, Karnataka, India

Nitesh Kumar: Department of Pharmacology, Manipal College of Pharmaceutical Sciences, Manipal University, Manipal 576104, Karnataka, India
Keywords: butter; miR-21; non-alcoholic fatty liver disease (NAFLD); non-alcoholic steatohepatitis (NASH); phosphatase and tensin homolog (PTEN); steatosis.

\section{Introduction}

In recent years, an increase in the incidence of obesity and diabetes mellitus (non-insulin dependent) has dramatically increased the incidence of non-alcoholic fatty liver disease (NAFLD), which results in non-alcoholic steatohepatitis (NASH) and which may develop further to hepatocellular carcinoma (HCC) [1]. Nowadays, NAFLD is considered as a social problem due to changes in lifestyle comprising increased intake of a fat-rich diet and the lack of exercise. As a result, more people are becoming obese with an increase in body mass index (BMI) and insulin resistance, which leads to the development of NASH [2]. Because of the asymptomatic behavior of the NAFLD, the condition remains unnoticed and without treatment for a long time, which leads to end-stage liver disease, i.e. NASH and requires liver transplantation to save patients [3]. Although genetic, epigenetic, and nutritional changes are also responsible for NAFLD, the recent increase in the incidence of NAFLD is mainly due to fatty acid overload among the general population.

To study pathogenesis and development of drugs, animal models, simulating the conditions of human disease, are always an important tool, which allow the investigator to study the pathological conditions of a disease and to identify the treatment strategy for that particular disease. To date, no satisfactory animal model is available to study NAFLD. The existing animal models either use genetically altered mice or mice with altered liver metabolism [4]. For instance, in altered liver metabolism, rats fed with choline-deficient (CD) and methionine choline-deficient (MCD) models are two animal models that are widely used in studies of NAFLD. In these models, NAFLD is induced due to the change in liver metabolism and does not involve obesity. Even in MCD-fed diet animals, weight loss with excess in hepatocellular injury is observed $[4,5]$, which truly differentiates it from the conditions associated with NAFLD observed in humans [6], 
while genetically altered animals do not show the same kind of disease progression. There were other attempts to create new models for studying NAFLD such as intra-gastric overnutrition [7], forced feeding of saturated fat-rich food to foz/foz mice [8], surgical implantation of gastrostomy tubings [7], sucrose and fructose overfeeding [7], etc. However, in the ideal animal model, the animal should develop steatohepatitis due to the feeding pattern simulating the behavior observed in humans [6], which was missing from the newly developed model.

Cell culture and animal models for alcoholic and nonalcoholic fatty liver disease cannot be easily simulated in animals as several factors are involved in the progression of these diseases [9]. However, a suitable in vitro model, which can be performed easily and rapidly at reduced cost and the one that is reproducible in animals, is essential before in vivo experiments. Besides, such in vitro techniques are useful in studying the pathogenesis involved in NAFLD and screening, drug candidates for hepatoprotective action against steatosis [10].

Thus, the present study was aimed to study the pathogenesis of NAFLD by the exposure of butter, a highly used fat in Chang liver cells using oleic acid as a control and the oil red O (ORO)-based colorimetric quantification method. Although attempts have been made to create NAFLD models in rats and chickens using a fat-rich diet [11, 12], it will be easier during a routine investigation if such models are created in the most commonly used Swiss albino mice fed with commonly used fat, i.e. butter-rich chow. Thus, the present study was designed to develop a new animal model of NAFLD by feeding chow rich in butter to Swiss albino mice. Furthermore, the exposed liver cells of animals were analyzed for PTEN and miR-21 expression. As the exposure of fat containing excess of unsaturated fatty acids to liver cells often causes PTEN deregulation/ downregulation through mTOR/NFjB-dependent mechanisms and involved in the development of NAFLD and other related malignancies such as NASH and HCC [13]. The downregulation of PTEN expression leading to increased invasiveness of hepatoma [14] has been particularly observed in the case of liver cells when compared to other cells, viz., breast and prostate [1]. Thus, the molecular study has been designed to validate the developed model through the PTEN expression pattern.

\section{Materials and methods}

All cell culture materials and media components were procured from Sigma-Aldrich (USA). Chang cells were procured from National Centre for Cell Sciences (Pune, India). Butter was purchased from Amul
(India). Rat chow was procured from VRK Nutritional Solutions (Pune, India). Other chemicals were of the highest grade and were procured from Merck (India).

\section{In vitro studies}

Oleic acid-induced steatosis: Chang liver cells were maintained in minimum essential medium (MEM) supplemented with 10\% fetal bovine serum (FBS) under standard cell culture conditions $\left(37^{\circ} \mathrm{C}\right.$, $5 \% \mathrm{CO}_{2}$ ). These cells were seeded into a 96-well flat bottom microtiter plate at a density of 20,000 cells per well and allowed to adhere by incubating at $37^{\circ} \mathrm{C}$ in $5 \% \mathrm{CO}_{2}$. The cells were supplemented with MEM with 10\% fetal bovine serum (FBS). When the cells reached $80 \%$ confluence, each well was treated with increasing concentration of oleic acid solution $(200 \mu \mathrm{L})$, viz., $0.25,0.5,0.75,1.0,1.25,1.5$, $1.75,2.0,4,8$, and $12 \mathrm{mM}$ for $24 \mathrm{~h}$. The experiments were performed in quadruplet. After $24 \mathrm{~h}$, the cells were examined for steatosis after removing the media.

Detection of steatosis: Accumulations of fatty acid within cells were examined using the oil red O method. Briefly, the cells were washed three times with doubled distilled water to remove traces of medium. The washed cells were fixed using $100 \mu \mathrm{L}$ of fixative solution containing $4 \%$ formaldehyde in phosphate-buffered saline (PBS) for $10 \mathrm{~min}$ at $37^{\circ} \mathrm{C}$. The fixative solution was removed and the cells were washed with double distilled water. The washed cells were stained with $50 \mu \mathrm{L}$ of $0.2 \%$ oil red $\mathrm{O}$ dissolved in isopropanol and volume adjusted with distilled water (ORO solution). The OROtreated cells were incubated at $37^{\circ} \mathrm{C}$ for $15 \mathrm{~min}$, after which the cells were washed with double distilled water until the solution became clear. This was followed by drying at $40^{\circ} \mathrm{C}$ for $30 \mathrm{~min}$ in a hot air oven. After drying, the cells were mounted with glycerin and examined under a microscope. Red oil droplets in the stained cells indicate oleic acid-induced steatosis.

Butter-induced steatosis: Chang liver cells, maintained as indicated previously, were seeded into 96-well flat bottom microwell plate at a density of 20,000 cells per well and cultured at $37^{\circ} \mathrm{C}$ in $5 \%$ $\mathrm{CO}_{2}$ atmosphere to allow for cell adhesion. After the cells attained $80 \%$ confluence, the cells were treated with varying concentrations of MEM medium containing melted butter. MEM $(1 \mathrm{~mL})$ containing $100 \mu \mathrm{L}$ of melted butter was considered as 100 dilutions. From this, the required MEM-butter mixtures, viz., $10^{-1}, 10^{-2}$, to $10^{-10}$, were prepared. The experiments were performed in quadruplet. Cells were examined for steatosis as described previously.

ORO-based steatosis quantification: Steatosis quantification using the ORO method was based on the Cui et al. [10] method. Steatosis was induced in Chang liver cells by oleic acid and butter. The cells were treated with $100 \mu \mathrm{L}$ of iso-propanol and incubated for $10 \mathrm{~min}$. ORO was allowed to be released from the cells by gently vibrating the plate. The released oil-ORO complex was transferred to another 96-well plate, and the optical density was measured at $405 \mathrm{~nm}$.

Preparation of fat-rich diet: Fat-rich diet was prepared using a rat/ mice pellet. The pellet was finely grounded and passed through the mesh (number 8) of $2.38 \mathrm{~mm}$. Marketed butter was mixed with this fine powder such that the final fat concentration was around $38 \% \mathrm{w} / \mathrm{w}$. 
This mixture was made into a small clump of $20 \mathrm{~g}$ each. This fat-rich diet was stored at $-20^{\circ} \mathrm{C}$ until used in the animal studies.

\section{In vivo studies}

A study was conducted after obtaining the approval from the Institutional Animal Ethics Committee (IAEC) of Kasturba Medical College (KMC), Manipal (No. IAEC/KMC/01/2012). Swiss albino mice (male) 2 months of age, were acclimatized to the experimental room having a temperature of $23 \pm 2^{\circ} \mathrm{C}$, controlled humidity conditions, and a 12:12-h light and dark cycle. Animals were caged in polypropylene cages with a maximum of four animals in each cage. The mice were divided in two groups. One group was fed with standard food pellet, i.e. without the addition of butter; the other group was fed with a specially formulated diet. Both groups were provided fresh water ad libitum. An initial pilot study was carried out for 1 week with four animals to check for the palatability of the specially formulated diet for the mice after which the main experiment was carried out. Forty-eight mice were divided into two main groups. Twenty-four mice were provided with the standard food pellets and water (control group). The other group was fed with the formulated diet and water (special diet group). These main groups were further subdivided into three subgroups consisting of eight animals in each subgroup. Each subgroup had one control and one special diet group. The feed was weighed and added to each cage in multiples of $20 \mathrm{~g}$ of feed per animal. All the animals were weighed regularly at an interval of 3-4 days. The feed remaining in the cages was also weighed to calculate the amount of food consumed. All data were recorded. The cages were changed every third day for maintenance. Animals from each group were sacrificed at the 35th, 70th, and 105th days. Blood was withdrawn retro-orbitally from the animals under light anesthesia; after which, the animals were sacrificed by cervical dislocation. Plasma from the blood mixed with EDTA was separated and stored at $-20^{\circ} \mathrm{C}$ until further processing. Livers removed from the sacrificed animals were divided into two halves. One-half was stored in $4 \%$ formaldehyde solution and another at $-80^{\circ} \mathrm{C}$ for further processing. The change in the animal weight and the average amount of feed/ special diet consumed were recorded regularly.

\section{Expression studies: RT-PCR analysis}

PTEN and miR-21 expression studies using reverse transcriptase-PCR analysis: RNA was extracted from the excised livers (100 mg) using TRIzol reagent (Invitrogen, USA) and quantified by UV spectrometry (A260/280 method). cDNA was synthesized from isolated RNA using Invitrogen's SuperScript ${ }^{\circledR}$ III First-Strand synthesis as per the manufacturer's protocol. DNA was synthesized from cDNA for PTEN and

Table 1: Primer sequences.

\begin{tabular}{ll}
\hline Primers & Sequence \\
\hline PTEN forward primer & -GAC AGC CAT CAT CAA AGA GAT CG \\
PTEN reverse primer & -GGT TCA TTC TCT GGA TCA GAG TCA GTG \\
miR-21 forward primer & -GTC GGG TAG CTT ATC AGA CTG \\
miR-21 reverse primer & -CAG ACA GCC CAT CGA CTG GTG \\
\hline
\end{tabular}

miR-21with respective primers using Invitrogen's KOD hot start DNA polymerase as per the manufacturer's protocol. RNA and PCR product were run on $1 \%$ agarose gel and stained with ethidium bromide, visualized, and documented under ultraviolet (Alpha Imager instrument). Sequences for primers used are given in Table 1.

\section{Statistical analysis}

All the data were expressed as mean \pm SEM. Data were analyzed by an unpaired t-test using GraphPad Prism demo version 5 for Windows (GraphPad Software, San Diego, CA, USA).

\section{Results}

\section{In vitro staining}

ORO staining clearly indicated the accumulation of fat as small droplets in a well-distributed manner throughout the cells. With increasing doses of oleic acid and butter, a progressive increase in the steatosis was observed (Figure 1). The butter-induced steatosis was comparable to that obtained with oleic acid and, hence, can be considered as a suitable substance to be used to induce a cell culture steatosis/NAFLD model.

\section{In vitro quantification}

The extent of fat accumulation within the cells was determined after quantifying the red color added to the cell by the ORO dye. The increase in absorbance reflected the increase in degree of steatosis. As the concentration of oleic acid increased from $0.25 \mathrm{mM}$ to $4 \mathrm{mM}$, there was a marked increase in absorbance, which reflected the increased fatty accumulation within the Chang liver cells (Figure 1). In the case of the butter-treated group, a linear increase in fat accumulation within the Chang liver cells was observed as the log concentration of butter was increased from $10^{-9}$ to 1 (Figure 1).

\section{In vivo studies}

\section{Correlation between fat-rich diet and weight gain}

Both special diet-treated mice and controls gained 4-5 g of weight. Though more significant weight gain and feed consumption was expected from the special diet, a lesser weight gain was observed. This insignificant difference 
Oleic acid induced steatosis
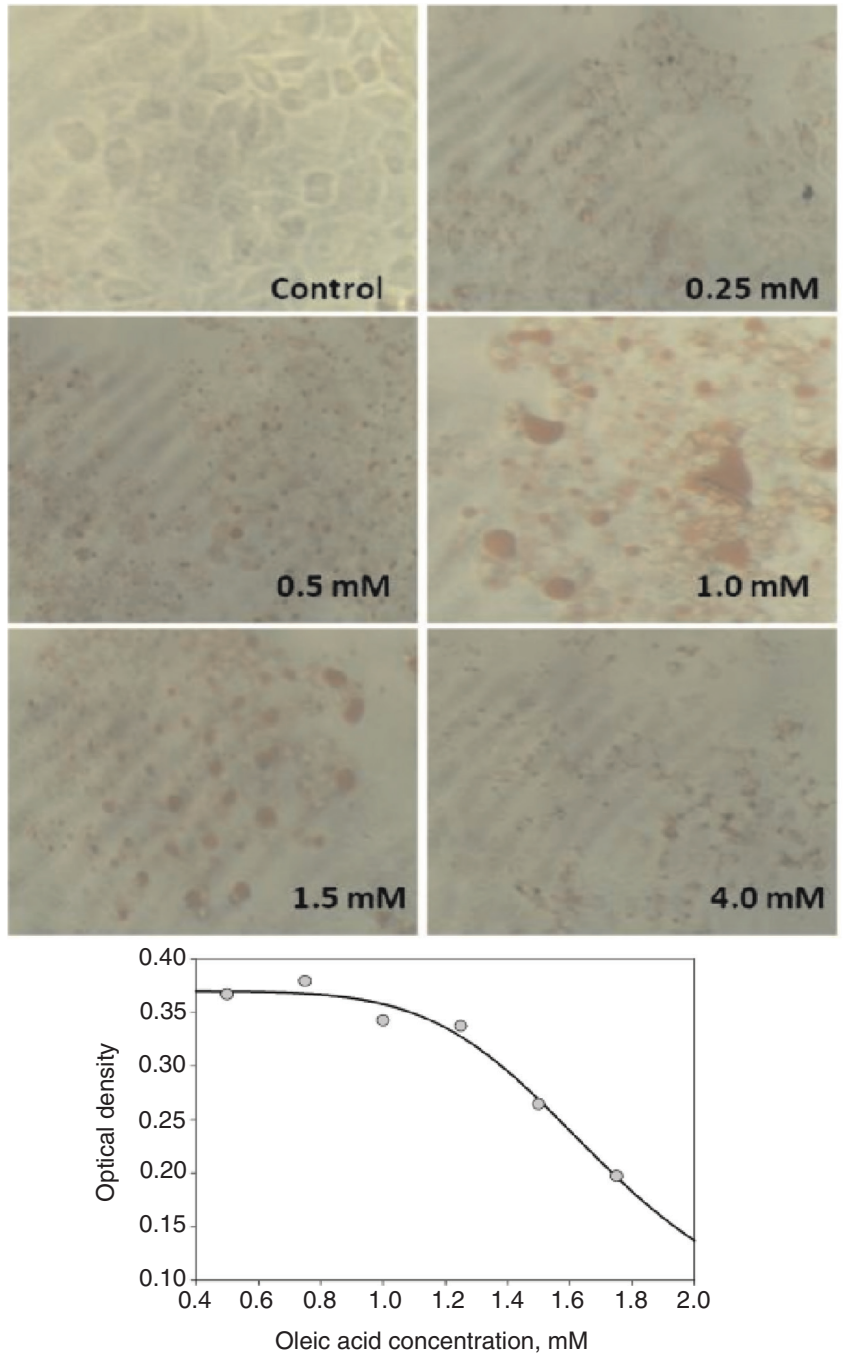

Butter induced steatosis
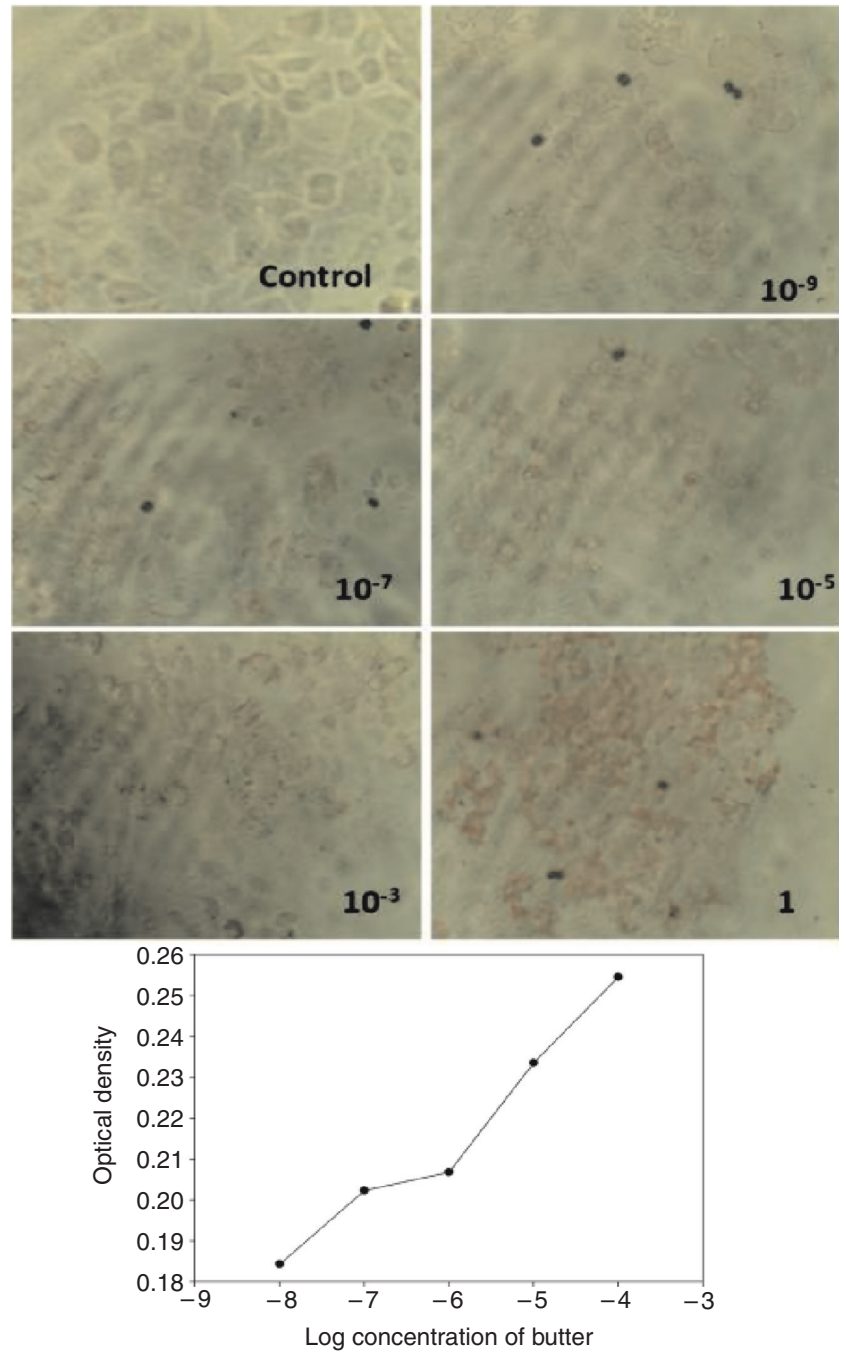

Figure 1: Oleic acid- and butter-induced steatosis in Chang liver cells.

As the oleic acid and butter concentrations increased, there was an increase in triglyceride accumulation, seen as red patches in the cell. As the concentration of oleic acid increased from $0.25 \mathrm{mM}$ to $4 \mathrm{mM}$ and the concentration of butter increased from $10^{-9}$ to 1 , a marked increase in the amount and extent of fat (stained red, with oil red 0 ) accumulation was observed.

in weight gain between the two groups could be due to the starting age of the animals, i.e. the animals were 2 months old at the time of the start of the experiment. Hence, all the animals had already reached nearly $30 \mathrm{~g}$ of weight resulting in insignificant weight gain (Figure 2). Further, as the special diet was not forced fed, test mice, due to the high fat content of the special diet, could have consumed proportionately less than its normal feeding habit on control diet. Hence, though the control group consumed nearly $7 \mathrm{~g}$ per animal per day, the special diet group animals consumed only around $3 \mathrm{~g}$ per animal per day. Moreover, the diet was introduced only in limited quantities of 40-80 g per visit to prevent spoilage and encourage feeding in mice.

\section{In vivo biochemical parameters}

Alanine aminotransferase (ALT), total and direct bilirubin levels in the special diet group were found to be significantly elevated $(p<0.05)$ compared to the control diet group after the 10th week onward. Alkaline phosphatase (ALP) level in the special diet group showed significant $(p<0.05)$ elevation from 5th week onward compared to the control group. Aspartate aminotransferase (AST) triglycerides were found to be significantly $(p<0.05)$ elevated on the 15th week. However, the level of direct bilirubin was more pronounced in the special group than in the control. Increased levels of these parameters are indicative of liver disease (Figure 3). 


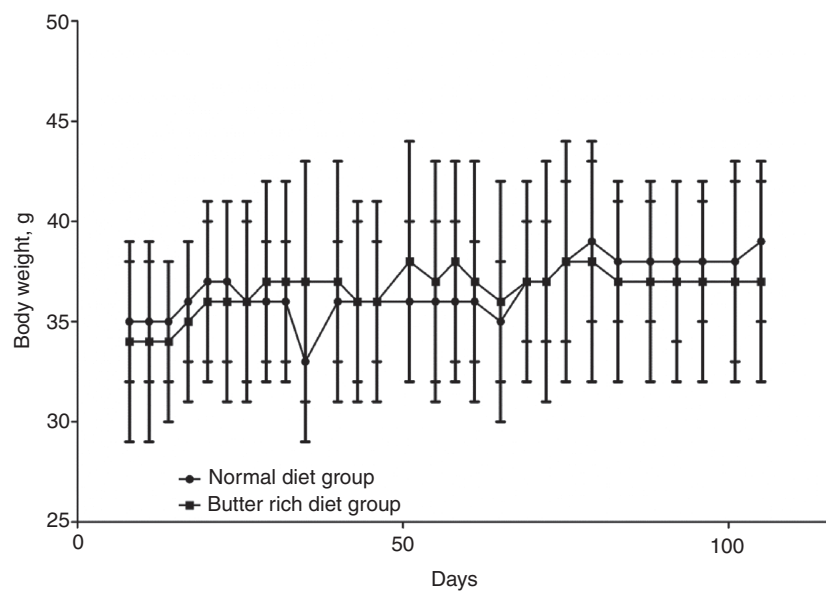

Figure 2: Body weight of animals.

Changes in the body weight of mice fed with normal and special diet over a period of 15 weeks.

\section{PTEN and miR-21 expression}

Gene expression studies showed that PTEN is downregulated in the special diet group compared to that in the control group. This indicates that the special diet used in this model is able to induce NAFLD and steatosis. After 10 weeks, the gene expression studies showed faint bands not consistent with any proposed theories. This was equal in both groups.

The third time period again showed a marginal but evident indication that PTEN was still downregulated compared to the control group. However, in the case of $m i R-21$, the levels of $m i R-21$ expression were almost similar in both groups (Figure 4).

\section{In vivo histopathology}

Histopathology evaluation of hepatocytes showed clumping, loss of shape and perivenular necrotic changes. Inflammatory changes, viz., hepatic ballooning and cytoplasmic aggregates were observed in the 15th week histology. This clearly indicates that the high-fat diet had initiated progression to NAFLD (Figure 5).

\section{Discussion}

Metabolic syndrome affecting liver has not received the same attention as is given to the other metabolic
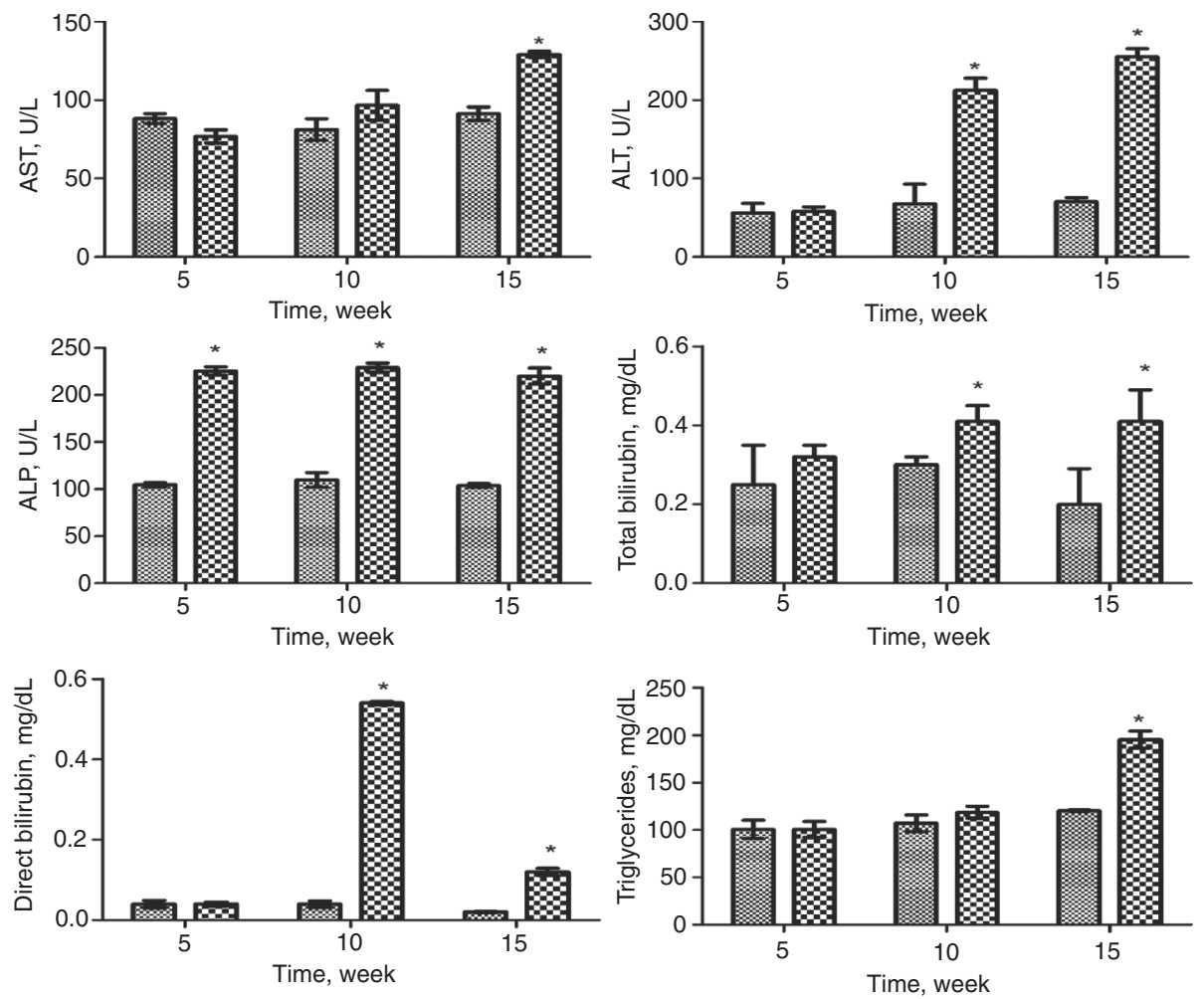

Normal diet

Butter rich diet

Figure 3: Biochemical estimation.

All the values are mean \pm SEM of six animals. Data are analyzed using the t-test where ${ }^{*} \mathrm{p}<0.05$ compared to controls. 


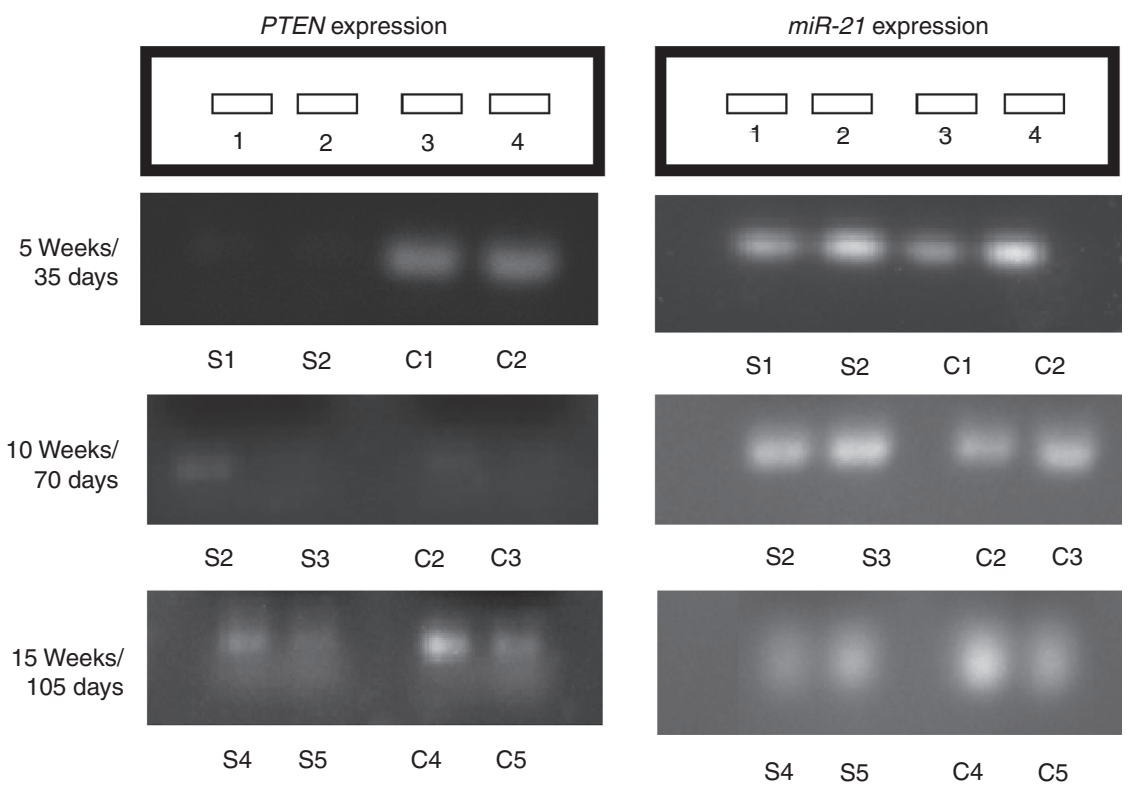

Figure 4: Expression of PTEN and miR-21.

While the test groups that were fed with special diet (S1 and S2) showed increased PTEN expression, there was no difference in the level of miR-21 expression compared to the control groups ( $\mathrm{C} 1$ and $\mathrm{C} 2)$ fed with normal diet.

syndromes, viz., diabetes and coronary heart disease. However, considering the growing incidence of NAFLD leading to hepatocellular carcinoma (HCC) there is an urgent need to develop treatment strategies, but this could be achieved only if suitable in vitro and in vivo animal models are created, which can replicate the conditions of NAFLD observed in the human population [4]. In the present study, the NAFLD was developed in cell lines and in mice using edible butter. Butter is one of the major ingredients present in fat-rich diets and is consumed throughout the world. It contains a mixture of fatty acids, viz., saturated and unsaturated fatty acids. The saturated fatty acid present in butter is generally converted to oleic acid. This oleic acid along with other long chain unsaturated fatty acids present in the butter could cause hepatic steatosis [15]. In the present study, the edible butterinduced steatosis in the cell line was compared with oleic acid-induced steatosis [10]. This kind of model will serve as a better indicator to study the progression of the disease and ultimately testing of newer treatment strategies.

For establishing, the butter-induced steatosis in in vitro, the concentration of butter was an important parameter. The concentration of butter used for the study was expressed as a log of its quantity, as it contains a number of components, which is difficult to quantify. Pure butter was given a value of 10 . Concentrations made subsequently from this would, therefore, represent $10^{-1}$, $10^{-2}$, etc. The steatosis induced by oleic acid and butter in Chang liver cells was assessed using ORO, a diazo dye, which stains neutral triglycerides and lipids. The accumulation of fatty acid within liver cells was seen as small red oil droplets distributed throughout the cells. The extent of steatosis induced in the cells depended on the dose of oleic acid and butter. After a certain concentration range, i.e. 0.5 and $1.75 \mathrm{mM}$ for oleic acid and $10^{-4}$ to $10^{-8}$ for butter, it showed the plateau phase for fatty accumulation, which might be due to the plateau phase of apoptosis as reported by Cui et al. [10] (Figures 3 and 5). Such a quantitative model can be useful in studying and correlating the effects of inflammatory mediators, apoptosis and stress with NAFLD. Even any other inducible factor of NAFLD can be identified and correlated to events within the quantifiable concentration range of that inducing agent.

In in vivo study, mice were fed with butter-rich special diet for 15 weeks. A diet rich in butter not only contains palmitic acid and oleic acid but also a mixture of various forms of fatty acids, which mimics the diet pattern of humans [11]. Studies showed that weight gain in the mice was only 4-5 g in both special groups as well as control diet groups. This might be because the animals were obtained at 2 months of age and had already reached nearly $30 \mathrm{~g}$ of weight. Furthermore, as the special diet was not forced fed, we can see that weight gain in the special diet group did not exceed that of the control diet. The other possible reasons might be due to the high fat content of the diet, which affected the feed intake. Though the control diet was consumed at nearly $7 \mathrm{~g}$ per animal per day, the 


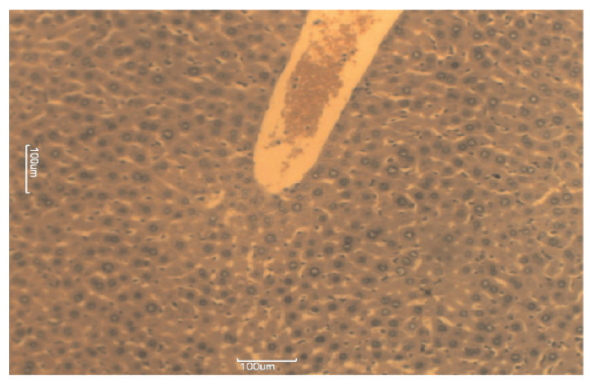

Control diet 5 week

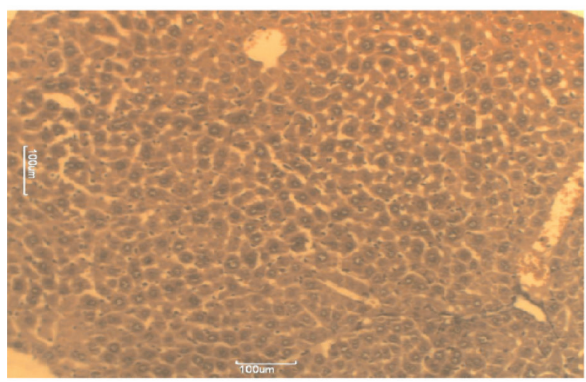

Control diet 10 week

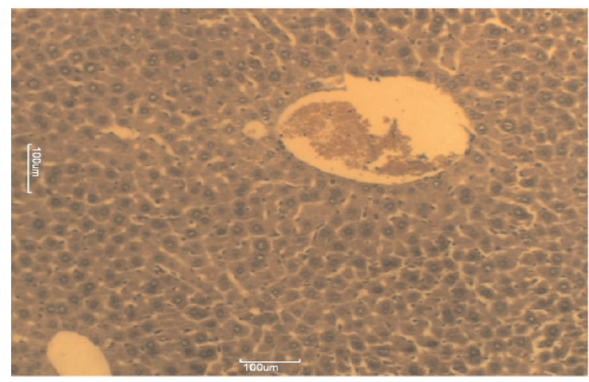

Control diet 15 week

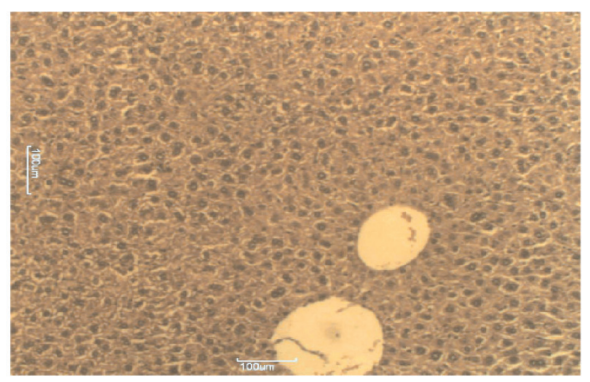

Special diet 5 week

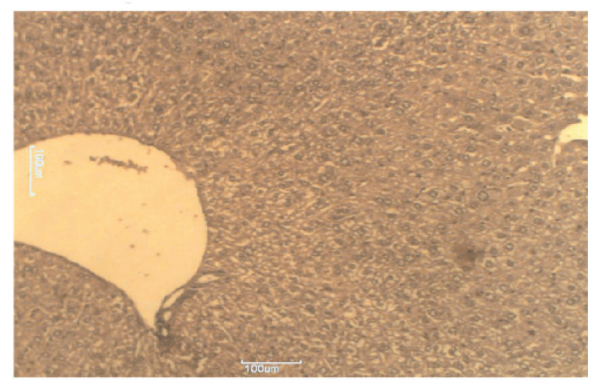

Special diet 10 week

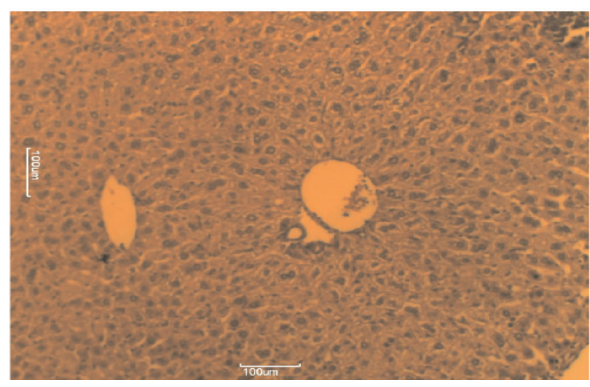

Special diet 15 week

Figure 5: Histopathology of liver from normal- and special diet-fed mice.

The figure shows the effects of the special diet on the liver hepatocytes such as necro-inflammatory changes, hepatocytes ballooning.

special diet was only at around $3 \mathrm{~g}$ per animal, but the weight changes in the groups were the same.

Steatohepatitis develops as a result of a "double hit". The presence of oxidizable fat within the liver leads to the development of steatosis, which is considered as the "first hit". The "first hit" is mainly due to excess fat accumulation and insulin resistance, which further increases the availability of free fatty acid. The free fatty acid is oxidized to aldehyde, viz., 4-hydroxynoneal and malondialdehyde (MDA), which are the main contributing factors for the development of Mallory bodies. Besides, these aldehydes activate NF- $\kappa B$, which are involved in the recruitment of various inflammatory cytokines and generating free radicals. These free radicals are considered as the "second hit”. Along with free radicals, decreased hepatic ATP turnover causes oxidative stress in the liver and finally leads to steatohepatitis $[9,16]$. In such steatohepatic conditions, lipogenic tissues such as the liver, which are involved in maintaining lipid homeostasis, are mostly affected due to free fatty acid-mediated downregulation of PTEN and may lead to hepatocellular carcinoma. Previously in vitro experiments to study the effect of unsaturated fatty acids on hepatic cells have been carried out on HepG2 and $\mathrm{HuH}-7$ cells [1]. PTEN is an anti-proliferative gene, which suppresses proliferation of hepatic carcinoma cells by suppressing the action of PI3K through dephosphorylation [17]. PTEN belongs to the protein tyrosine phosphatase families that are expressed constitutively in the cell. Downregulation of PTEN often leads to increased cell proliferation and invasion. PTEN is inactivated in the presence of reactive oxygen species such as hydrogen peroxide due to the formation of disulfide linkages between the cysteine residues at its active site. Type I phosphoinositide 3-kinases (PI 3) are activated upon phosphorylation. These kinases regulate the number of cellular activities including cell survival, growth, proliferation, 
and motility in response to insulin. PTEN antagonizes PI 3-kinases through its phosphatase action and regulates its activities [18]. After this, disorder further detoriates to HCC either through cirrhosis or directly without involving cirrhosis. Excess unsaturated free fatty acids suppress PTEN expression. The present study showed a similar trend in the downregulation of PTEN.

$\mathrm{miR}$, which comprises a group of small nucleotides (19-25 in length), is involved in negative regulation of genes and plays an important role in HCC through direct suppression of PTEN expression. Subtype, miR-21 acts mainly by activating focal adhesion kinase (FAK) and Akt through phosphorylation, while PTEN acts by involving dephosphorylation of $F A K$ in controlling cell migration. Thus, upregulation of miR-21 will directly indicate an increased malignancy condition [19]. However, in the current study, there was no significant difference in the miR-21 level between the control and test groups, which might be due to the short duration of the study. The study was not continued until the development of HCC, which might be the reason for the unchanged level of miR-21. In addition, the other cofactor such as diabetes was not induced. However, it is important to ascertain that as $m i R$-21 levels have been proposed to increase in the NAFLD pathology, the ratio between miR-21 and PTEN levels will also increase, which is more important than their quantitative increase. Hence, a fall in PTEN levels alone would clearly disturb the balance, and $m i R-21$ will be greater relative to the amount of PTEN present. Thus, the observed downregulation of PTEN and unchanged miR21 indicates that NAFLD progression was started.

Normally, in the NAFLD, the degree of inflammation and fibrosis are confirmed by liver biopsy. Typically macro vascular steatosis, lobular inflammation, hepatocytes necrosis, Mallory bodies, fibrosis, and cirrhosis are observed in NAFLD histology [20]. In the present study, the histology and biochemical parameters were considered as an essential factor for the assessment of the endpoints of a special high-fat diet. These tests confirmed that special diets had initiated progression to NAFLD. Excess lipids were stored as large vesicles displacing the nucleus resulting in a signet ring appearance. These lipids were reported to be triglycerides, a neutral lipid by Donato et al. 2007 [15]. This macro vesicular steatosis is an important hallmark of NAFLD, which occurs due to the increased influx but reduced efflux of free fatty acid. It is observed in obesity and diabetes-induced steatosis. Steatosis due to drug toxin or genetic condition results in impaired $\beta$-fatty acid oxidation and results in microvesicular hepatic steatosis with no nuclear displacement of the nucleus [21]. We could not find the symptom of NASH as the dietary intake of mice were under their control. Even earlier reports suggested that when rats were fed with a fat-rich diet both in liquid and solid forms, only those rats fed with highfat liquid diet progressed to NASH even though both the groups developed steatosis [22].

The biochemical estimations in serum samples showed a significant elevation of LFT parameters, i.e. AST, ALT, ALP direct and total bilirubin levels in the butter-rich diet groups. Elevation in these parameters was a clear indication of liver disease. An increase in triglyceride levels reflected an increase in imbalance in lipid metabolism, which was supported by a histopathology study. Thus, after analyzing the biochemical parameters, histopathological markers, and expression study, it can be concluded that NAFLD was induced by a butter-rich diet. Prolonged treatment and adding some more hits may lead to NASH.

However, studying NAFLD on a short duration model in mice would not be completely justifiable considering that the duration of the disease progression in normal patients often takes years. Considering the short lifespan of the rodents, it would be better to consider inducing more stress factors than a high-fat diet alone to induce "hits" that would trigger a faster progression of disease in the liver.

Acknowledgments: We would like to thank the Manipal College of Pharmaceutical Sciences, Manipal, and Manipal University for providing the research facility. The financial support from DST-FIST is gratefully acknowledged.

Author contributions: All the authors have accepted responsibility for the entire content of this submitted manuscript and approved submission.

Research funding: Department of Science and Technology, Ministry of Science and Technology.

Employment or leadership: None declared.

Honorarium: None declared.

Competing interests: The funding organization(s) played no role in the study design; in the collection, analysis, and interpretation of data; in the writing of the report; or in the decision to submit the report for publication.

\section{References}

1. Vinciguerra M, Carrozzino F, Peyrou M, Carlone S, Montesano R, Benelli R, et al. Unsaturated fatty acids promote hepatoma proliferation and progression through downregulation of the tumor suppressor PTEN. J Hepatol 2009;50:1132-41.

2. Chavez-Tapia NC, Rosso N, Tiribelli C. In vitro models for the study of non-alcoholic fatty liver disease. Curr Med Chem 2011;18:1079-84. 
3. Rolo AP, Teodoro JS, Palmeira CM. Role of oxidative stress in the pathogenesis of nonalcoholic steatohepatitis. Free Radic Biol Med 2012;52:59-69.

4. Schattenberg JM, Galle PR. Animal models of non-alcoholic steatohepatitis: of mice and man. Dig Dis 2010;28:247-54.

5. Rinella ME, Elias MS, Smolak RR, Fu T, Borensztajn J, Green RM. Mechanisms of hepatic steatosis in mice fed a lipogenic methionine choline-deficient diet. J Lipid Res 2008;49:1068-76.

6. Larter CZ, Yeh MM. Animal models of NASH: getting both pathology and metabolic context right. J Gastroenterol Hepatol 2008;23:1635-48.

7. Deng QG, She H, Cheng JH, French SW, Koop DR, Xiong S, Tsukamoto H. Steatohepatitis induced by intragastric overfeeding in mice. Hepatology 2005;42:905-14.

8. Arsov T, Larter CZ, Nolan C J, Petrovsky N, Goodnow CC, Teoh NC, et al. Adaptive failure to high-fat diet characterizes steatohepatitis in Alms1 mutant mice. Biochem Biophys Res Commun 2006;342:1152-9.

9. Day CP, James OF. Steatohepatitis: a tale of two "hits"? Gastroenterology 1998;114:842-5.

10. Cui W, Chen SL, Hu KQ. Quantification and mechanisms of oleic acid-induced steatosis in HepG2 cells. Am J Transl Res 2010;2:95-104.

11. Omagari K, Kato S, Tsuneyama K, Inohara C, Kuroda Y, Tsukuda $\mathrm{H}$, et al. Effects of a long-term high-fat diet and switching from a high-fat to low-fat, standard diet on hepatic fat accumulation in Sprague-Dawley rats. Dig Dis Sci 2008;53:3206-12.

12. Ayala I, Castillo AM, Adánez G, Fernández-Rufete A, Pérez BG, Castells MT. Hyperlipidemic chicken as a model of non-alcoholic steatohepatitis. Exp Biol Med (Maywood) 2009;234:10-6.

13. Watanabe S, Horie Y, Kataoka E, Sato W, Dohmen T, Ohshima $S$, et al. Non-alcoholic steatohepatitis and hepatocellular carcinoma: lessons from hepatocyte-specific phosphatase and tensin homolog (PTEN)-deficient mice. J Gastroenterol Hepatol 2007;22(Suppl 1):S96-100.

14. Vinciguerra M, Veyrat-Durebex C, Moukil MA, Rubbia-Brandt L, Rohner-Jeanrenaud F, Foti M. PTEN down-regulation by unsaturated fatty acids triggers hepatic steatosis via an NFkappaBp65/mTOR-dependent mechanism. Gastroenterology 2008;134:268-80.

15. Gómez-Lechón MJ, Donato MT, Martínez-Romero A, Jiménez N, Castell JV, O'Connor JE. A human hepatocellular in vitro model to investigate steatosis. Chem Biol Interact 2007;165:106-16.

16. Serviddio G, Sastre J, Bellanti F, Viña J, Vendemiale G, Altomare E. Mitochondrial involvement in non-alcoholic steatohepatitis. Mol Aspects Med 2008;29:22-35.

17. Downes CP, Perera N, Ross S, Leslie NR. Substrate specificity and acute regulation of the tumour suppressor phosphatase, PTEN. Biochem Soc Symp 2007:69-80.

18. Downes CP, Walker S, McConnachie G, Lindsay Y, Batty IH, Leslie NR. Acute regulation of the tumour suppressor phosphatase, PTEN, by anionic lipids and reactive oxygen species. Biochem Soc Trans 2004;32(Pt 2):338-42.

19. Meng F, Henson R, Wehbe-Janek H, Ghoshal K, Jacob ST, Patel T. MicroRNA-21 regulates expression of the PTEN tumor suppressor gene in human hepatocellular cancer. Gastroenterology 200;133:647-58.

20. Russo MW, Jacobson IM. Nonalcoholic fatty liver disease. Hosp Physician 2002;67:36-41.

21. Reddy JK, Rao MS. Lipid metabolism and liver inflammation. II. Fatty liver disease and fatty acid oxidation. Am J Physiol Gastrointest Liver Physiol 2006;290:G852-8.

22. Gaemers IC, Stallen JM, Kunne C, Wallner C, van Werven J, Nederveen A, et al. Lipotoxicity and steatohepatitis in an overfed mouse model for non-alcoholic fatty liver disease. Biochim Biophys Acta 2011;1812:447-58. 